\title{
Clínica de Riesgo Obstétrico
}

\author{
Hospital Universitario del Valle. Cali. Colombia
}

Carlos A. Quintero J., M.D.* Jaime Rodríguez, M.D.**

Ramiro Muñoz***

\section{DEFINICION Y JUSTIFICACION DE LA CLINICA DE EMBARAZO DE RIESGO OBSTETRICO}

La Clínica de Embarazo de Riesgo Obstétrico funciona a partir del mes de noviembre de 1975 como una clínica especial ambulatoria del Hospital Universitario del Valle. Su creación fue la respuesta a la demanda de pacientes obstétricas con riesgo materno que de acuerdo a normas específicas eran en primera instancia atendidas en centros de salud, hospitales locales o regionales y remitidas al Hospital Universitario del Valle. Además, como una política del Departamento de Obstetricia y Ginecología tendiente a desarrollar los aspectos

* Profesor Asociado. Jefe del Departamento de Obstetricia y Ginecología. Universidad del Valle. Cali. Colombia.

* Profesor Asociado. Departamento de Medicina Social. Universidad del Valle. Cali. Colombia.

*** Ing. de Sistemas. Investigador Asociado. Departamento de Medicina Social. Universidad del Valle. Cali, Colombia. tecnológicos y ofrecer una calidad de atención propia de su nivel.

Inicialmente fue incluida en la consulta prenatal general del Hospital y luego se identificó como consulta de embarazo de riesgo, desapareciendo la consulta prenatal normal en la institución.

EI H.U.V. es la única institución de salud del Departamento del Valle que cuenta con una infraestructura locativa de personal y recursos tecnológicos para funcionar dentro de un programa de atención materna como nivel terciario.

\section{RECURSO HUMANO Y TECNOLOGICO}

La clínica cuenta con 3 especialistas quienes han tenido experiencia perinatal previa, un Ph D en Medicina Perinatal, un especialista con entrenamiento en endocrinología y 2 residentes de úl timo año del Departamento de Obstetricia y Ginecología. El personal anterior es equivalente a 3 tiempos completos (40 hs. $x$ semana), dedicados a la clínica to el resto del personal se refiere al de salud de Consul ta Externa: 2 enfermeras y 4 auxiliares de enfermería. 
Desde el punto de vista tecnológico se cuenta con un laboratorio de medicina perinatal con 3 tecnólogas de laboratorio de tiempo completo entrenadas en monitoría bioquímica y biofísica ante e intraparto.

Se ofrece un servicio de consulta de 6 horas semanales y un servicio de laboratorio de 12 horas diarias, 5 días a la semana. Los exámenes que realiza, además de los de rutina son:

Glicemia pre, post-prandial y curva de tolerancia oral a la glucosa.

Líquido amniótico: celularidad, prueba de Clements, delta de densidad óptica a 450 y $650 \mathrm{~m} \mu$, espectrofotometría, relación lecitina-esfingomielina, bilirrubina, exámen directo y cultivo y cariotipo.

T3 - T4 - TSH e índice de tiroxina libre, título de anticuerpos anti $\mathrm{Rh}$, estriol en plasma y orina, $\mathrm{pH}$ de sangre fetal (cuero cabelludo).

\section{Biofísicos:}

Monitoría anteparto: prueba de respuesta de la frecuencia cardíaca fetal (FCF) a los movimientos fetales (NST) y prueba de respuesta de la FCF a las contracciones (OCT o Prueba de Posse).

Monitoría intraparto: técnicas directa con electrodo de cloruro de plata para FCF y catéter para actividad uterina $(A \mu)$.

Ultrasonido: ecografía de tiempo real y estática $(1,2)$.

\section{DESCRIPCION DEL FUNCIONAMIENTO}

De acuerdo a la figura No. 1, toda paciente que ingresa a la clínica debe tener una remisión; luego se realiza un diagnóstico y una clasificación de riesgo, de acuerdo a normas establecidas y se decide una conducta de hospitalización - control ambulatorio. Las pacientes con riesgo bajo se remiten de nuevo a

\section{Figura No. 1}

FLUJOGRAMA DE ATENCION EN LA CLINICA DE EMBARAZO DE ALTO RIESGO H. U. V.

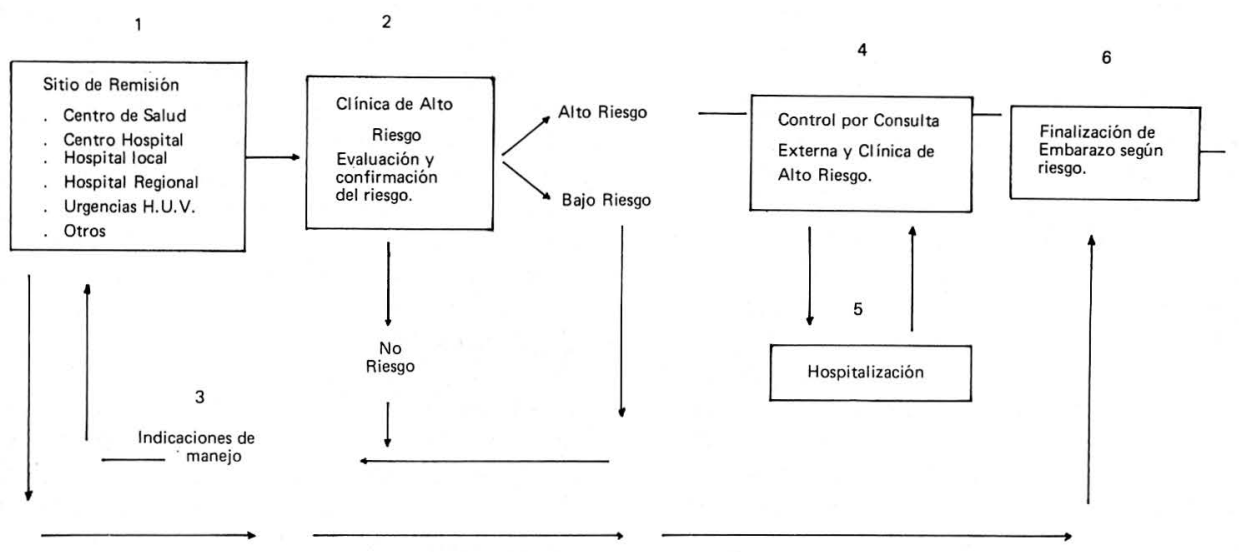


un nivel primario y secundario para continuar su control de acuerdo a las normas pertinentes en dichos niveles.

\section{NORMAS DE ATENCION DE RIESGO OBSTETRICO}

Se han utilizado las normas tradicionales de los Servicios de Medicina Perinatal y Riesgo Obstétrico, las cuales en la actualidad se están revisando con el fin de elaborar los manuales propios del departamento $(3,4,5,6)$.

\section{CRITERIOS DE RIESGO}

El servicio no utiliza instrumentos de clasificación de riesgo con un valor ponderado de las diferentes variables sino que cada uno de los factores se analiza en forma individual usando como referencia los índices de otras clínicas $(7,8)$. Además de los factores de riesgos que se relacionan con el individuo, por ejemplo: edad, estado civil, escolaridad, ingresos, etc., se tienen en cuenta los siguientes:

Antecedentes personales médicos: diabetes, TBC, enfermedad hipertensiva, cardiopatía, síndrome convulsivo, in-compatibilidad Rh, enfermedad previa, etc.

Antecedentes Familiares: Diabetes, malformaciones congénitas, embarazo múltiple y otras.

Antecedentes Ginecoístétricos: Gravidez, paridad, número de cesáreas, abortos, embarazos extrauterinos y molares, hijos vivos actuales, número de mortinatos, muertes neonatales, prematuros y niños con malformaciones. Datos referentes a la terminación del embarazo anterior, parto espontáneo, inducido, operación cesárea y otras intervenciones como fórceps y vacuum.
Patología del embarazo anterior como toxemia, hemorragias, presentaciones distócicas.

Diagnóstico realizado en el primer control o durante la evolución: Incompatibilidad $\mathrm{Rh}$, síndrome convulsivo, infertilidad previa, polihidramnios, lúes, toxoplasmosis, diabetes, anemias, cardiopatías, infección urinaria, embarazo gemelar, distocias, enfermedad tiroidea, enfermedad hipertensiva, toxemia, hemorragias, amenaza de parto prematuro, embarazo prolongado, RPM, y otras.

Los criterios anteriores se manejan de acuerdo con su severidad y un buen porcentaje de las pacientes se podrían referir a niveles de atención secundaria, si tuvieran una estructura adecuada. Sin embargo, en lo referente a la atención del parto está definido que sea en el H.U.V.

\section{ANALISIS DE LA CLINICA DURANTE EL PERIODO DE ENERO/76-DICIEMBRE/80}

Al revisar las historias clínicas de las pacientes atendidas se obtuvieron 1.100 casos, de los cuales se descartaron 65 $(6 \%)$, por no corresponder a embarazos de riesgo y 273 (25\%), cuyas historias no pudieron ser localizadas en el archivo. Es decir, se analizan las características del embarazo de 762 mujeres (69\%). En 144 casos, se desconoce el resultado final del embarazo (3\%) debido a que el parto fue atendido en sitio distinto al Hospital Universitario y no fue posible obtener la información pertinente.

\section{Cobertura de la Clínica:}

Teniendo en cuenta que el HUV es una institución de referencia y de atención especializada, la población objetivo de la clínica sería el porcentaje de embarazos de riesgo, estimados en base al 
número de nacidos vivos y mortinatos, descartando los embarazos atendidos por el Seguro Social y la práctica privada. Los datos de Bayona y Rico (9) en el período de estudio, muestran para Cali 119.047 niños vivos y ajustando esta cifra con un $2 \%$ de mortinatos habrían finalizado 121.428 embarazos, de los cuales son asignables al Sistema Nacional de Salud, aproximadamente un 60\%, es decir, 72.856 embarazos. Suponiendo un máximo de 20.6 de embarazo de riesgo, se tendría una cobertura* de la clínica de aproximadamente $7.3 \%$.

Por supuesto, la cifra anterior es solamente un estimativo, pero se calcula para mostrar la importancia de utilizar oportunamente dicha información $y$ estimular acciones de salud coordinadamente entre los distintos niveles de atención.

\section{Características de las embarazadas atendidas en la clínica}

El 10\% tienen menos de 20 años $y$ el $21.4 \%$ más de 35 años, es decir, en una de cada tres mujeres, la edad fue un factor de riesgo. El primer sitio de referencia a la clínica es el propio hospital universitario, con 600 casos (78.8\%). Esto puede ser explicado porque las referencias de la periferia son atendidas en Urgencias y luego enviadas, según el caso, a la clínica. Figuran como referidas de centros de salud $y$ hospitales 140 (18.4\%). Es importante anotar que solo 12 pacientes aparecen referidas fuera de Cali

\section{Antecedentes:}

Tienen antecedentes personales positivos 326 pacientes $(42.7 \%$ y familiares

\footnotetext{
* 220 emb. alto riesgo, atendidos por año, en HUV,
}

345 (45.3\%). En el Cuadro No. 1, se calcula cada antecedente con relación al total de embarazos (762).

\section{Cuadro No. 1}

ANTECEDENTES PERSONALES

$Y$ FAMILIARES DE EMBARAZADAS

ATENDIDAS EN LA CLINICA DE RIESGO.

1976 - 1980 H.U.V. - CALI

\begin{tabular}{|llllll|}
\hline \multicolumn{2}{|l|}{ Personales } & & & \\
\hline Diabetes & $3.14 \%$ & Hipertensión & $12.3 \%$ & S. Convulsivo & $4.1 \%$ \\
TBC & $1.84 \%$ & Cardiopatia & $7.6 \%$ & Incompatib. Rh. & $10 . \%$ \\
\hline Familiares & & & & \\
\hline Diabetes & $15.2 \%$ & Malf. Cong. & $2.2 \%$ & Emh. Múltiple & $27.8 \%$ \\
\hline
\end{tabular}

El embarazo anterior al actual terminó en parto espontáneo en 380 casos (49.8\%) y $12.2 \%$ terminaron por operación cesárea. Estos datos implican una intervención en la terminación del embarazo anterior, por cada dos partos. Por otra parte, este dato se desconoce en 141 casos (18.5\%).

Los antecedentes ginecobstétricos se distribuyen así:

$$
\text { 응 }
$$

Gravidez: $\quad$ uno 16.3 cinco y más: 31.2 Paridad: cero 33 cinco y más: 14.2 Cesáreas: una 11 dos y más: 3.9

Abortos: $\quad$ uno- 31.7 tres y más: $\quad 8.5$ dos

Mortinatos: uno 10.9 dos y más: 3.9

Prematuros: uno 6.4 dos y más: 2.4

Malf. Cong; una 1.7 dos: 0.2

El 7.2 (55) tenían antecedentes de muertes neonatales en los primeros 7 días y el 3\% (20) muertes entre 7 y 28 días. 
Presentaron anormalidades en el embarazo anterior 283 mujeres (37.1\%), distribuidas así: toxemia $18.2 \%$, hemorragia $4.6 \%$ distocia pélvica $4.8 \%$, presentación distócica $5.2 \%$, ruptura prematura de membranas $4.2 \%$.

\section{Evolución del embarazo}

En el primer control se hace diagnóstico de riesgo por antecedentes ginecobstétricos en 236 (19.1\%) y por entidades específicas en 998 (80.9\%) para un total de 1234 diagnósticos en las 762 embarazadas.

Las principales entidades se muestran en el Cuadro No. 2 (incluye primero o segundo diagnóstico).

Cuadro No. 2

DIAGNOSTICOS REALIZADOS EN

EL PRIMERO O SEGUNDO CONTROL

\begin{tabular}{|l|c|}
\hline Diagnóstico & $\frac{\%}{}$ \\
\hline Incompatibilidad Rh & 16.4 \\
Toxemia & 10.6 \\
Enfermedad hipertensiva & 10.2 \\
Cardiopat ías & 8.8 \\
Infección urinaria & 8.1 \\
Amenaza parto prematuro & 6.4 \\
Diabetes & 5.0 \\
Lúes & 2.9 \\
Síndrome Convulsivo & 2.9 \\
Distocias & 2.2 \\
\hline
\end{tabular}

\section{Exámenes de laboratorio}

Los exámenes de laboratorio realizados en la clínica fueron los siguientes:
Examen

No. de

Realizados

Hemoglobina

Parcial de orina

666

Clasificación RH

607

Serología

601

Glicemia

Citología vaginal

Coombs indirecto

Líquido amniótico para amb. fetal

Cultivo de orina

Prueba no stress

Prueba respuesta a oxitocina

Estriol

50

$R x$. tórax

Líquido amniótico para Rh

Antic. Rubeola

Ecografía

T3

T4

Cariotipo

En general, el $\mathrm{Rh}$, la serología y el parcial de orina, son los exámenes más frecuentes. Se realizaron cuatro exámenes por paciente (2576), de los cuales fueron patológicos el $35.8 \%$.

En algunos casos los exámenes pedidos o no fueron realizados o no se consignó en la historia el resultado. Además, por limitaciones del hospital o razón económica del paciente, exámenes tales como estrioles, cariotipo de líquido amniótico, ecografía y aún la misma 
monitoría electrónica de la FCF, no fue posible obtenerla.

\section{Hospitalización:}

De las 162 mujeres se hospitalizaron durante el embarazo 106 (14\%) por las siguientes razones: falla tratamiento $0.8 \%$, por complicaciones específicas $11.8 \%$ y para métodos de diagnóstico el $1.3 \%$

\section{Control prenatal:}

El número de controles prenatales por paciente fue: uno, $24.4 \%$, dos, 22.5\%; tres, 14.5\%; cuatro, 14.7\%; de cinco a nueve, 22.3\%; y diez y más controles el $1 \%$. Esto implica un promedio de 3.2 controles por embarazada $y$ aproximadamente el $50 \%$ de las mismas tuvieron sólo dos controles después de su ingreso a la clínica.

La edad gestacional al último control y al término del embarazo, se muestra en el Cuadro No. 3.

\section{Atención del parto:}

Fueron atendidas en el Hospital Universitario 622 pacientes $(81.6 \%)$, un caso en el Seguro Social, 8 en Centros de Salud. En el $17.2 \%$ se desconoce el sitio y las características del parto, por lo tanto la información se dará solamente sobre los partos atendidos en el Hospital Universitario del Valle.

\section{Forma de terminación del embarazo:}

$\begin{array}{ll}74.1 \% & \text { Parto vaginal } \\ 25.9 \% & \text { Cesáreas } \\ 16.4 \% & \text { Parto inducido }\end{array}$

\section{Patología fetal:}

Vivo (548) $88.7 \%$, vivo con sufrimiento (52) $8.4 \%$ y muerto (20) $3.2 \%$ de 618 partos.

Mortalidad fetal: $32.3 \%$

\section{Patología ovular:}

Normal, $80.9 \%$; ruptura prematura de membranas, $18.1 \%$ Abruptio placentae $0.5 \%$ y placenta previa $0.5 \%$.

\section{Complicaciones maternas:}

Ninguna: $\quad 74.8 \%$

Cuadro No. 3

EDAD GESTACIONAL AL ULTIMO

CONTROL Y AL TERMINAR EL EMBARAZO CLINICA DE ALTO RIESGO

1976 - 1980. HUV.CALI

\begin{tabular}{|c|c|c|c|c|c|c|}
\hline & \multicolumn{5}{|c|}{ Semanas de gestación } & Total \\
\hline & $<20$ & $21-30$ & $31-35$ & $36-40$ & $41 y+$ S. D. & \multirow{3}{*}{762} \\
\hline $\begin{array}{l}\text { Ultimo control } \\
\left(\frac{\circ}{0}\right)\end{array}$ & 4.7 & 8.4 & 19.9 & 65.2 & $2.7-$ & \\
\hline $\begin{array}{c}\text { Termino } \\
\text { embarazo \% }\end{array}$ & 1.0 & 1.8 & 4.1 & 65.1 & $10.6 \quad 17.3$ & \\
\hline
\end{tabular}


$25.2 \%$ las siguientes:

$\begin{array}{lr}\text { Ruptura uterina } & 1 \\ \text { Eclampsia } & 46 \\ \text { Distocias } & 21 \\ \text { Hemorragia post parto } & 7 \\ \text { Choque } & 1 \\ \text { Sepsis } & 3 \\ \text { Desgarros menores } & 67 \\ \text { Otras } & 12\end{array}$

No hubo muertes maternas de las 762 historias revisadas.

\section{Complicaciones del recién nacido:}

Ninguna: $94.4 \%$

$5.6 \%$ las siguientes:

SDR (moderado y severo)13

Broncoaspiración $\mathbf{5}$

Sepsis

Meningocele

Enf. Hem. del R. N. 1

OTRAS

as

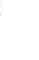


Cuadro No. 6

EMBARAZADAS CON Y SIN TOXEMIA SEGUN RESULTADO FINAL CLINICA DE RIESGO 1976 - 1980. HUV. CLOMBIA

\begin{tabular}{|c|rrrr|r|}
\hline \multirow{2}{*}{ TOXEMIA } & \multicolumn{3}{|c|}{ PATOLOGIA FETAL } & \multirow{2}{*}{ TOTAL } \\
\cline { 2 - 5 } & Muerto & Vivo + Suf. & Vivo & S. D. & \\
\hline Si (\%) & 5.0 & 9.0 & 72.7 & 13.1 & 99 \\
No (\%) & 2.0 & 6.5 & 81.8 & 19.8 & 663 \\
\hline
\end{tabular}

\begin{tabular}{|c|ccc|c|}
\hline \multirow{2}{*}{ TOXEMIA } & \multicolumn{2}{|c|}{ COMPLICACIONES DEL RECIEN NACIDO } & \multirow{2}{*}{ TOTAL } \\
\cline { 2 - 3 } & SI & NO & S.D. & \\
\hline Si $(\%)$ & 26.3 & 60.6 & 13.1 & 99 \\
No $(\%)$ & 15.5 & 64.8 & 19.6 & 663 \\
\hline
\end{tabular}

\begin{tabular}{|l|rcr|r|}
\hline \multirow{2}{*}{ TOXEMIA } & \multicolumn{3}{|c|}{ PESO AL NACER } & \multirow{2}{*}{ TOTAL } \\
\cline { 2 - 4 } & $<2500$ & $2500 y+$ & S.D. & \\
\hline Si (\%) & 15.2 & 64.6 & 20.1 & 99 \\
No (\%) & 10.6 & 66.0 & 23.4 & 663 \\
\hline
\end{tabular}

\begin{tabular}{|c|cccc|c|}
\hline \multirow{2}{*}{ TOXEMIA } & \multicolumn{4}{|c|}{ APGAR AL MINUTO } & \multirow{2}{*}{ TOTAL } \\
\cline { 2 - 5 } & 0 & $1-7$ & 8 y + & S.D. & \\
\hline Si (\%) & 5.0 & 21.2 & 58.6 & 15.1 & 99 \\
No (\%) & 2.3 & 14.3 & 62.3 & 21.1 & 663 \\
\hline
\end{tabular}

\begin{tabular}{|c|cccc|c|}
\hline \multirow{2}{*}{ TOXEMIA } & \multicolumn{4}{|c|}{ APGAR A LOS 5 MINUTOS } & \multirow{2}{*}{ TOTAL } \\
\cline { 2 - 5 } & 0 & $1-7$ & $8 y+$ & S. D. & \\
\hline Si (\%) & 7.0 & 7.0 & 70.7 & 15.1 & 99 \\
No (\%) & 2.3 & 4.2 & 72.2 & 21.3 & 663 \\
\hline
\end{tabular}


Se aplicó la prueba estadística $\mathrm{Chi}^{2}$ para variables independientes en las diferentes tablas y el análisis dejó en claro que en cada una de ellas se debe aceptar la hipótesis de independencia, es decir, que las variables no se relacionan entre sí. Lo anterior se puede explicar posiblemente debido a la falta de datos (38\% en todo el estudio).

\section{COMENTARIOS Y RECOMENDACIONES}

1. El análisis de un problema de atención materna como es el embarazo de riesgo obstétrico, lleva implícita la necesidad de crear diferentes niveles de atención que permitan responder a la demanda de una patología materno fetal, la cual es responsable de la mortalidad perinatal y buena parte de la materna. Es así comolos centros universitarios deben tener una infraestructura que permita dar una cobertura adecuada a la demanda, basándose en normas de atención definidas $y$ contando con los recursos tecnológi$\cos y$ humanos necesarios.

2. Hemos visto que es muy importante definir criterios de riesgo que permitan a un servicio identificar $y$ clasificar en forma ágil y adecuada, el riesgo que tiene una mujer embarazada $y$ cómo evolucionará éste a través de su embarazo. Independientemente del uso de instrumentos que permitan dar una asignación de un determinado riesgo, la normatización de la atención debe basarse en una serie de criterios que hagan establecer prioridades de atención de acuerdo a la severidad de los hallazgos, tanto por los antecedentes como por la evolución de un determinado embarazo, para obtener un buen resultado.

3. Las instituciones a las cuales les corresponde dar una atención de nivel terciario deben contar con los recursos adecuados es decir, una infraestruc- tura básica, una tecnología minima pero funcionante $y$ un personal dedicado exclusivamente a este tipo de atención.

De la misma manera debe existir un sistema que permita al paciente un fácil acceso a los servicios. Como se vio en el análisis de la clínica de nuestro hospital, hay dificultad para realizar exámenes básicos, necesarios para la adecuada atención y el seguimiento de los embarazos de riesgo. Además el control prenatal debe ser normatizado de acuerdo a cada caso en particular y en lo posible detectar tempranamente los embarazos para clasificar su riesgo.

4. Debe existir una sistematización de la información que permita realizar un análisis adecuado de los eventos con el fin de efectuar evaluaciones periódicas de calidad y eficiencia y analizar en un período determinado el resultado final con el objeto de modificar índices.

5. Desde el punto de vista administrativo, debe realizarse un estudio de factibilidad que permita identificar los problemas relacionados con remisión de pacientes, atención oportuna $y$ adecuada, análisis de costos, etc. y pueda planearse en base a los resultados, un programa de atención de riesgo materno fetal.

\section{SUMARIO}

Se hizo una revisión de la Clínica de Riesgo Obstétrico referente a su funcionamiento, recursos humanos y tecnológicos $y$ criterios de riesgo. Se revisaron 1.100 historias clínicas que correspondieron a los primeros 5 años. Se descontaron $30 \%$ por dificultades de archivo o error de clasificación del diagnóstico. Se analizaron las características, antecedentes $\mathrm{y}$ evolución del embarazo 
con sus correspondientes diagnósticos, así como los exámenes de laboratorio realizados. Se hace una descripción de la patología materna, fetal y del recién nacido. Se intentó hacer un cruce entre patología obstétrica y el resultado del embarazo y el recién nacido, con el fin de buscar dependencia entre las variables pero posiblemente debido a que la muestra era incompleta no pudo encontrarse. A manera de ejemplo se hizo lo anterior, para pacientes que presentaron toxemia. Finalmente, se hacen los comentarios y las recomendaciones pertinentes al funcionamiento de una Clínica de Riesgo Obstétrico haciendo énfasis en la planificación de la misma desde el punto de vista operacional administrativo, tecnológico y el uso de información sistematizada que permita evaluar permanentemente con el fin de efectuar acciones en salud.

\section{OBSTETRICAL RISK CLINIC - HOPSITAL UNIVERSITARIO DELVALLE}

A summary of the High Risk outpatient Clinic with regard to its function, human resources, technology and risk criteria was made. 1.100 clinical records from the first 5 years were analyzed, out of this number, $30 \%$ were excluded due to diverse difficulties. Different characteristics, antecedents, evolution of pregnancy with its corresponding diagnosis and laboratory exams were analyzed. A description of maternal, fetal and neonatal pathology was

\section{BIBLIOGRAFIA}

1. EFFER, S.B. Biochemical and biophysical indice of fetal risk. Clinics in Perinatology 1 (1): 161-172 (1974).

2. PAUL R. PETRIC R. Fetal intensive care, University of S. California, 1979.

3. BUTLER, W.R. High risk predictors at booking and in pregnancy. Perinatal problems. 1969. pp. 36-46.

4. HOBEL, C.J. Prenatal and intrapartum highrisk screeining. II. Risk factors reassessed. Am. J. of Obstetrics and Gynecology, 135: 1051-1056 (1979).

5. WEISS, W., JACKSON, E. Maternal factor affecting birth weight. Perinatal factor affecting human development. PAHO. 1969 pp. 54-59. done, and in addition, a correlation between obstetrics pathology and the outcoming result of the pregnancy and newborn was tried, to look for a direct relationship between the variables but the result was not significant. $\mathrm{Fi}$ nally, comments and recomendations pertinent to the functionality of the High Risk out-patient Clinic in order to obtain better results in perinatal mortality and morbidity were made.

6. AUBRY, R. NESBITT, R. High Risk Obstetrics I. Perinatal outcome in relation to a broadened approach to obstetric care for patients at special risk. Am. J. of Obstetrics and Gynecol. 105 (2) 241-247, 1969.

7. EDWARDS, L.E. A simplified antepartum risk acoring system. Obstetrics and Gynecology 54 (2): $237-240$ (1979)

8. SOKOL, R. Clinical application of highrisk scoring on an obstetric service. Am. J. Obstetrics and Gynecology 128 (6) 652 661 (1977).

9. BAYONA, A., RICO, J. El descenso de la fecundidad y su impacto sobre la estructura de la mortalidad en la ciudad de Cali. CCRP. Implicaciones socio-económicas y demográficas del descenso de la fecundidad en Colombia. Vol. 18, abril/82. 